\title{
SEIS AÑOS DE EXPERIENCIA EN CIRUGÍA LAPAROSCÓPICA LUMBAR EXTRAPERITONEAL: INDICACIONES, COMPUCACIONES Y RESULTADOS EN UN CENTRO IBEROAMERICANO DE REFERENCIA
}

\author{
M arcos Tobias-M achado, M arco Túlio Lasmar, Freddy Rincón Ríos, Pedro Hermínio Forseto, \\ Roberto Vaz Juliano y Eric Roger W roclawski.
}

Disciplina de Urología, Faculdad de Medicina de ABC. Santo André. São Paulo. Brasil.

\begin{abstract}
Resumen.- O BJETIVO : Presentar la experiencia en cirugía laparoscópica lumbar extraperitoneal obtenida con el perfeccionamiento de la técnica durante un período de 6 años.

MÉTO DO S: Se realizó un estudio prospectivo con 168 casos operados entre 1999 y 2004 a tra vés de cirugía laparoscópica con acceso extraperitoneal para diversa s pa to logías reno-ureterales y adrenales. Las cirugías fueron subdivididas de acuerdo al órgano comprometido y a la complejidad del procedimento (ablativo o reconstrutivo). Se describieron el número de casos operados, tiempo quirúrgico, índice de complicaciones mayores y menores, número de conversiones para acceso convencional, período de hospitalización,
\end{abstract}

M arcos Tobias-M achado

Rua G raúna, N.o 104, Ap 13

M oema, São Paulo-SP

CEP: $04514-000$. Brasil.

tobias-machado@uol.com.br

Trabajo recibido: 30 de febrero 2004 tiempo de retorno a las actividades habituales, las ventajas y desventajas, así como tambien análisis de acuerdo con los datos de la literatura.

RESULTADO S: El acceso extraperitoneal fue empleado en 168 cirugías laparoscópicas, siendo 44 biopsias renales, 8 marsupializaciones de quiste renal, 49 nefrectomías (22 por enfermedades benignas y 27 por neoplasias), 15 nefroureterectomías, 22 adrenalectomías, 15 pielolito/ ureterolitotomías, 1 nefropexia, 2 nefrectomías parciales, 11 pieloplastias y 1 corrección de ureter retrocavo. El tiempo quirúrgico se redujo significativamente después del período de adaptación inicial, con un promedio de 110 min para 138 cirugías ablativas y de 163 min para 30 procedimientos reconstructivos. Hubo apenas una conversión en el 2.0 caso operado. La tasa de complicaciones mayores y menores fue de $5,07 \%$ y $4,34 \%$ respectiva mente para el total de cirugías ablativas y de $3,33 \%$ y $6,66 \%$ para el total de cirugías reconstrutivas. El tiempo promedio de hospitalización global varió de 1 a 4 días. El retorno a las actividades habituales se dio en un promedio de 7 a 30 días de postoperatorio, de acuerdo a la complejidad de la cirugía.

CONCLUSIONES: El acceso laparoscópico lumbar extraperitoneal es una excelente opción de abordaje mínimamente invasivo para cirugías ablativas, presentando las ventajas al no violarse la cavidad peritoneal, con bajo índice de complicaciones. Las cirugías reconstrutivas son factíbles, pero técnicamente más difíciles, dependiendo de la habilidad y adaptación del cirujano a un menor espacio de trabajo. 
Palabras clave: Laparoscopía. N efrectomía laparoscópica. Adrenalectomía. Pieloplastía laparoscópica. Espacio retroperitoneal.

Summary.- O BJEC TIVES: To report the experience in lumbar extraperitoneal laparoscopy surgery obtained while perfectioning the technique over a six-year period.

METHODS: W e perform a prospective study with 168 cases of extra peritoneal laparoscopic surgery for the treatment of various kidney-ureter and adrenal diseases between 1999 and 2004. O perations were classified by organ and complexity (ablative or reconstructive). W e describe the number of cases, surgical time, mayor and minor complications, number of open conversions, hospital stay, time to return to daily-life activities, advantages and disadvantages, as well as comparative analysis with data from bibliography.

RESULTS: Extraperitoneal access was employed in 168 laparoscopic operations: 44 renal biopsies, 8 renal cyst marsupializations, 49 nephrectomies $(22$ benign diseases/ 27 neoplasias). 15 nephroureterectomies. 22 adrenalectomies.15 pyelolithotomies/ ureterolithotomies, 1 neprhropexy, 2 partial nephrectomies. 11 pyeloplasties and 1 correction of retrocaval ureter. 0 perative time decreased significant only after the initial adaptation, with an average of 118 minutes in 138 ablative operations and 163 minutes in 30 reconstructive procedures. There was only one conversion in the second case of the series. The incidence of mayor and minor complications was $5.07 \%$ and $4.34 \%$ respectively for ablative surgery and $3.33 \%$ and $6.66 \%$ for reconstructive surgery. Average hospital stay varied from 1-4 days. Return to daily life activities to ok an average of 7 to 30 days in relation with procedure complexity.

CON CLUSION S: Lumbar extra peritoneal laparoscopic access is an excellent option of minimally invasive approach to ablative surgery, offering the advantages of avoidance of the peritoneal cavity and low complication index. Reconstructive surgery is feasible but technically more challenging, depending of the ability and adaptation of the surgeon to a smaller surgical workspace.

Keywords: Laparoscopy. Laparoscopic nephrectomy. Adrenalectomy. Laparoscopic pyeloplasty. Retroperitoneum.

\section{INTRODUCCIÓN}

La cirugía laparoscópica ha sido considerada el estandard de oro en el tratamiento de las enfermedades reno-ureterales y de las glándulas suprarrenales en lo referente a procedimientos ablativos (1).

Sin embargo, no existe concenso con respecto a los accesos para la técnica laparoscópica en Urología (2).

La mayoría de los autores han preferido el acceso transperitoneal por la facilidad de manipulación de los trócares y de la localización anatómica de las estructuras, principalmente por un mayor espacio de trabajo, de especial importancia en los procedimientos reconstrutivos (3).

Pocos trabajos en la literatura latino-americana han sido reportados empleando la técnica laparoscópica retroperitoneal exclusiva (4-8). Se tuvo por objetivo relatar una casuística de 168 casos de cirugías lumbares realizadas por retroperitoneoscopía, obtenida mediante el perfeccionamiento de esta técnica en un período de 6 años. Se realizaron comentarios relativos a los diversos procedimientos, enfatizando las ventajas y desvantajas de éste acceso, analizandolos de acuerdo con los datos de la literatura.

\section{MATERIALES Y MÉTODOS}

Durante el período de 1999 a 2004 se operaron 168 pacientes por 2 cirujanos donde se optó por el abordaje retroperitoneoscópico en procedimientos ablativos $(n=138)$ y reconstructivos $(n=30)$ del tracto urinario superior y adrenal. Las cirugías realizadas fueron: 44 biopsias renales, 8 marsupializaciones de quiste renal, 49 nefrectomías (27 radicales y $22 \mathrm{sim}$ ples), 15 nefroureterectomías (10 radicales y 5 simples), 22 adrenalectomías, 11 pieloplastías, 15 pielolitotomías/ ureterolitotomías, 1 nefropexia, 2 nefrectomías parciales y 1 corrección de ureter retrocavo.

Se utilizó el acceso estandard con obtención del espacio a través de disección digital asociada al uso de balón pneumático adaptado. $(4,9)$

Las cirugías fueron subdivididas de acuerdo al órgano comprometido y a la complejidad del pro- 
cedimiento (ablativo o reconstrutivo), siendo descritos el número de casos operados, número de trócares empleados, tiempo quirúrgico, índice de complicaciones mayores y menores y su tratamiento, número de conversiones para acceso convencional, período de hospitalización y tiempo de retorno a las actividades normales. Se definió como complicaciones mayores las situaciones que generaban necesidad de transfusión sanguínea peri-operatoria, conversión de emergencia o complicaciones que prolongaban el período de hospitalización o provocaban daño en la recuperación funcional. Las demás se consideraron complicaciones menores. Los registros obtenidos fueron recogidos prospectivamente a través de análisis de datos pre-operatorios, tiempo de hospitalización y seguimiento ambulatorio, el cual fue realizado rutinariamente con un protocolo de 7,30 y 60 días de postoperatorio.

\section{RESULTADOS}

\section{Cirugías ablativas (Tabla I)}

\section{Biopsia renal}

$\mathrm{N}$ uestras indicaciones iniciales para el acceso retroperitoneoscópico fueron los casos de biopsia renal. Se realizaron 44 casos, comenzando los 4 primeros a través de 3 trócares y los demás con 2 trócares. La curva de aprendizaje fue verificada a través del tiempo quirúrgico que, inicialmente era de 150 minutos, alcanzando luego de 10 casos un tiempo promedio de 45 [30 - 150] minutos. En 3 casos hubo la abertura accidental del peritoneo, pero sin ocasionar dificultades técnicas al cirujano. 0 currió apenas una conversión en esta serie, en el segundo caso, donde debido al aprendizaje inicial, el riñón no pudo ser visualizado con exactitud.

TABLA I. DISTRIBUCIÓN DE LOS CASOS O PERADOS POR ACCESO LAPARO SC Ó PICO EXTRAPERITO N EAL SIG UIEN DO CARACTERISTICAS TÉCN ICAS Y EVO LUCIÓN Q UIRÚRG ICA.

CIRUG IAS ABLATIVAS.

\begin{tabular}{|c|c|c|c|c|c|c|c|c|}
\hline CIRUGIAS & $\mathrm{N} \cong$ & $\begin{array}{c}\mathrm{N} 0 \\
\text { Trócares }\end{array}$ & $\begin{array}{l}\text { T quir. (promed)- } \\
\min \end{array}$ & $\begin{array}{l}\text { Compl. } \\
\text { Mayores }\end{array}$ & $\begin{array}{l}\text { Compl. } \\
\text { Menores }\end{array}$ & Conversiones & $\begin{array}{c}\text { Días de } \\
\text { Hospitaliza. } \\
\text { (promedio) }\end{array}$ & $\begin{array}{l}\text { Retorno a las } \\
\text { actividades } \\
\text { normales } \\
\text { (promed)-días }\end{array}$ \\
\hline Biopsia renal & 44 & $3 / 2$ & $30-150(45)$ & 1 & 0 & 1 & $0,5-2(1)$ & $5-8(7)$ \\
\hline $\begin{array}{l}\text { Marsupialización } \\
\text { quiste renal }\end{array}$ & 8 & 3 & $40-150 \quad(60)$ & 0 & 0 & 0 & $1-2(1)$ & $10-21(15)$ \\
\hline \multicolumn{9}{|l|}{ Nefrectomía } \\
\hline Simple & 22 & 4 & 40-190(85) & 0 & 1 & 0 & $1-4(2)$ & $10-21(15)$ \\
\hline Radical & 27 & 4 & $90-240(150)$ & 3 & 2 & 0 & $1-4(3)$ & $15-30(21)$ \\
\hline Nefroureterectomía & 15 & 4 & $150-300(200)$ & 2 & 2 & 0 & $3-6(4)$ & $21-40(30)$ \\
\hline Adrenalectomía & 22 & 4 & $110-180(135)$ & 1 & 1 & 0 & $1-21(3)$ & $10-35(15)$ \\
\hline TOTAL & 138 & & & $\begin{array}{c}7 \\
(5,07)\end{array}$ & $\begin{array}{c}6 \\
(4,34 \%)\end{array}$ & $\begin{array}{c}1 \\
(0,72 \%)\end{array}$ & & \\
\hline
\end{tabular}


La mayor parte de los pacientes fueron tratados con régimen de hospital-día y el retorno a las actividades rutinarias, se obtuvo en promedio al 7.0 día post-operatorio [5 a 10 días].

\section{Marsupialización de quiste renal}

La indicación de acceso laparoscópico para abordaje de quistes renales sigue las mismas indicaciones terapeúticas de la técnica convencional: compresión de la vía excretora, infección o hemorragia. Por esta patología, fueron intervenidos 8 casos (2 parapiélicos posteriores y 6 corticales), con diámetros variando entre 8 a $15 \mathrm{~cm}$, obteniéndose éxito en todos los casos en un seguimiento promedio de 15 meses. El acceso fue realizado a través de 3 trócares en todos los casos. Es importante disecar bien los límites del quiste antes de drenarlo, para que se tenga una perfecta orientación con relación a los bordes de tejido renal normal. La marsupialización fue realizada próxima al tejido renal normal para evitar recidiva del quiste y sus bordes fueron cauterizados. La recuperación de los pacientes fue excelente, sin presentarse complicaciones. El promedio de tiempo quirúrgico fue de 60 [40-150] minutos, el de la permanencia hospitalaria de 1 [1-2] día, y el de retorno a las actividades habituales de 15 [10-21] días posterior a la cirugía.

\section{N efrectomía}

Se intervinieron quirúrgica mente 22 pacientes con enfermedades benignas siendo los principales diagnósticos: litíasis o pionefrosis (12 casos), hipertensión arterial sistémica renovascular (3 casos), hidronefrosis y exclusión funcional renal (7 casos); y 27 casos de enfermedad renal maligna (tumores estadio T1-T2 de 4 a $10 \mathrm{~cm}$ ).

El plano de disección entre el peritoneo y la fascia de Gerota es muy delgada, ocurriendo en ocasiones, abertura peritoneal. La perforación peritoneal reduce el espacio de trabajo, pero promueve mayor seguridad en algunos casos adheridos en cuanto al correcto plano a ser disecado. En los últimos 15 casos no ocurrieron aberturas peritoneales.

En los casos de enfermedad benigna, la presencia de etiología inflamatoria (12 casos) promovió mayor dificuldad técnica traducida por un mayor tiempo promedio operatorio ( $70 \times 140 \mathrm{~min})$. El período de hospitalización varió de 1 a 4 días. En dos pacientes con diagnóstico anatomo-patológico de pionefrosis, se presentó infección de la pared abdominal que fue resuelto con drenaje y antibioticoterapia.

La cirugía radical siguió los mismos principios que la cirugía convencional, presentando un tiempo quirúrgico promedio de 150 [90 - 240] minutos y un tiempo promedio de hospitalización un poco mayor que la nefrectomía simple [3 $\times 2$ días]. En todos los casos fueron usados 4 trócares. En estos pacientes con tumor renal, a pesar de existir controversias, fue utilizada la retirada intacta del espécimen quirúrgico a través de la ampliación de uno de los trócares de 10 mm o por incisión de Pfannestiel.

En esta serie ocurrieron 2 lesiones vasculares controladas laparoscopicamente y 1 pneumotórax que ameritó de drenaje en el post-operatorio inmediato. A demás ocurrió un hematoma de retroperitoneo tratado conservadoramente y un caso de hérnia incisional post-operatoria. En seguimiento promedio de 24 meses, uno de los pacientes con tumor PT3 de alto grado presentó recidiva sistémica de la enfermedad. No se confirmó en este seguimiento ningún caso de implante tumoral en las incisiones. No hubo tampoco conversiones. Los pacientes retornaron a sus actividades normales en un promedio de 15 [10-21] días posterior a la cirugía para los casos de nefrectomías simples, a excepción de los casos de etiología inflamatoria, que requirieron un período promedio de retorno a las actividades semejante a los que fueron sometidos a nefrectomía radical - 21 [15-30] días.

\section{N efroureterectomía}

Q uince pacientes fueron sometidos a nefroureterectomía, siendo 5 casos de riñón con infección y reflujo, en pacientes de programas de transplante renal y 10 casos de carcinoma transicional de pelvis renal 0 ureter. Se emplearon también 4 trócares y los comentarios técnicos siguen los mismos utiliza dos para nefrectomía. La extracción de ureter distal fue obtenida por la intusucepción ureteral en 9 casos y a través de incisión de Gibson de $7 \mathrm{~cm}$ en otros 6 casos $(6,7)$. El tiempo quirúrgico promedio fue de 200 [150 - 300] minutos y el promedio de hospitalización de 4 [3 - 6] días.

Como complicaciones hubo un caso de fístula urinaría en 4.0 día post-operatorio en un paciente con incisión de $\mathrm{G}$ ibson, dos casos de hematoma retroperitoneal con evolución satisfactoria y un caso de morta- 
lidad por broncopneumonía y sepsis por germen nosocomial. En este caso ocurrió un tiempo quirúrgico mas prolongado, factor que pudo haber contribuído a una menor expansibilidad pulmonar. El tiempo transcurrido hasta retomar las actividades normales fue en promedio de 30 [21 - 40] días posterior a la cirugía.

\section{Adrenalectomía}

Realizamos 22 casos de adrenalectomía, incluyendo 3 casos de feocromocitoma, posterior a la experiencia adquirida con cirugía laparoscópica transperitoneal de la adrenal. La cirugía fue realizada a través de 4 trócares con tiempo promedio de 135 [110 - 180] minutos, sin complicaciones intra-operatorias. El promedio de tiempo post-operatorio en el hospital fue de 3 [1 - 21] días y el regreso a las actividades rutinarias se dio en promedio de 15 [10 - 35] días posterior a la cirugía. Uno de los pacientes (niño de 1,5 años) evolucionó con pneumonía en el post- operatorio lo que prolongó más su recuperación y el promedio global de hospitalización (8), y un paciente presentó hematoma de pared. Los demás casos presentaron buena evolución.

\section{Cirugías reconstrutivas (Tabla II)}

La necesidad de suturas en cirugía laparoscópica extraperitoneal aumenta significativamente el grado de dificuldad de la cirugía. Fueron realizados 30 procedimientos, siendo 11 pieloplastías (7 por la técnica de Fenger, 3 desmembradas y 1 pielo-ureterostomía látero-lateral), 15 casos de pielolitotomía / ureterolitotomia proximal, 1 nefropexia, 1 anastomosis uretero-ureteral para corrección de ureter retrocavo y 2 nefrectomías parciales. En todos los casos fueron utilizados 4 trócares, excepto para la corrección de ureter retrocavo que fue realizado con 3 trócares.

TABLA II. DISTRIBUCIÓN DE LOS CASOS O PERADOS PO R ACCESO LAPARO SC Ó PIC O EXTRAPERITO N EAL SIG UIEN DO CARACTERISTICAS TÉCN ICAS Y EVO LUCIÓN Q UIRÚRG ICA.

CIRUG IAS REC O N STRUTIVAS.

\begin{tabular}{|c|c|c|c|c|c|c|c|c|}
\hline CIRUGIAS & $\mathrm{N} \cong$ & $\begin{array}{c}\mathrm{N} 0 \\
\text { Trócares }\end{array}$ & $\begin{array}{l}\text { T quir. (promed)- } \\
\text { min }\end{array}$ & $\begin{array}{l}\text { Compl. } \\
\text { Mayores }\end{array}$ & $\begin{array}{l}\text { Compl. } \\
\text { Menores }\end{array}$ & Conversiones & $\begin{array}{c}\text { Días de } \\
\text { Hospitaliza. } \\
\text { (promedio) }\end{array}$ & $\begin{array}{c}\text { Retorno a las } \\
\text { actividades } \\
\text { normales } \\
\text { (promed)-días } \\
\end{array}$ \\
\hline $\begin{array}{l}\text { Pielolitotomía/ } \\
\text { Ureterolitotomía }\end{array}$ & 15 & 4 & $150-200(180)$ & 0 & 2 & 0 & $3-5(4)$ & $15-30(21)$ \\
\hline Nefropexia & 1 & 4 & 150 & 0 & 0 & 0 & 2 & 30 \\
\hline $\begin{array}{l}\text { Nefrectomía } \\
\text { parcial }\end{array}$ & 2 & 4 & $150-200(175)$ & 0 & 0 & 0 & 3 & 21 \\
\hline $\begin{array}{l}\text { Corrección de } \\
\text { ureter retrocavo }\end{array}$ & 1 & 3 & 130 & 0 & 0 & 0 & 2 & 21 \\
\hline Pieloplastia & 11 & 4 & $120-280(180)$ & 1 & 0 & 0 & $2-7(3)$ & $15-30(21)$ \\
\hline TOTAL & 30 & & & 1 & 2 & 0 & & \\
\hline & & & & $(3,33 \%)$ & $(6,66 \%)$ & & & \\
\hline
\end{tabular}


La técnica de Fenger es simple y factíble, con tiempo promedio de duración de 150 minutos. La cirugía desmembrada es mas trabajosa y el tiempo quirúrgico promedio en esta experiencia preliminar fue de 260 minutos. A penas un caso presentó fístula urinaría mas prolongada, pero con resolución espontánea manteniendose doble J y dren de Penrose. El estudio radiológico y cintilográfico de control evidenció el éxito de la cirugía en todos los pacientes. Uno de los pacientes sometidos a la técnica de Fenger persistió con dolor lumbar después de la cirugía. El período promedio de internación fue de 3 [2 - 7] días y el retorno a las actividades habituales ocurrió en un promedio de 21 [15 - 30] días.

En uno de los casos de pieloplastía y en la paciente con ureter retrocavo, fue realizada la exteriorización de los extremos ureterales para confección de la anastomosis de forma extracorporea.

En los casos de pielolitotomía fue constante la presencia de peripielitis intensa hasta que se identificó la pelvis y el ureter. La retirada de cálculo se realizó sin irregularidad en todos los casos, con tiempo quirúrgico promedio de 180 [150 - 200] minutos. En virtud de la friabilidad de la pelvis y de la hipercarpnia en el trans-operatorio, se optó por no suturar la misma en 2 pacientes, dejandose por seguridad apenas el cateter doble J, y retirandose éste posterior a las 6 semanas. El extravasamiento urinario fue auto-limitado con resolución total de la litiásis en todos los casos. El período promedio de internación hospitalaria y de retorno a las actividades rutinarias fueron respectivamente de 4 [3 - 5] y 21 [15 - 30] días.

La disección renal en el caso de nefropexia fue bastante simple. Hubo dificuldad en la aplicación del punto polar superior, debido a la angulación extrema de los trócares para alcanzar la musculatura posterior. El tiempo operatorio fue de $150 \mathrm{~min}$ y el de hospitalización de 2 días. No hubo complicaciones y el paciente retornó a las actividades luego de 30 días después de la cirugía. Posterior al seguimiento de 12 meses se comprobó mejoría de los síntomas y buen posicionamiento renal.

En dos casos de tumores exofísticos en la cara posterior del riñón con indicación de nefrectomía parcial, el acceso extraperitoneal con 4 trócares fue utilizado con éxito. Se realizó el reparo del pedículo renal con cinta cardíaca exteriorizada por uno de los trócares y la incisión del parénquima renal con margen de seguridad, fue realizado empleando bisturí ultrasónico. En los dos casos fue necesaria la compresión temporaria de la artería renal y sutura hemostática del parénquima renal. El promedio del tiempo quirúrgico fue de 175 [150 - 200] minutos. No se presentaron complicaciones o necesidad de transfusión sanguínea y el alta hospitalaria ocurrió al 3.0 día post-operatorio en ambos casos. Fue confirmado el diagnóstico de carcinoma renal de bajo grado con márgenes libres de neoplasia en los 2 casos y los pacientes retornaron a las actividades habituales a los 21 días después de la cirugía.

\section{DISCUSIÓN}

La cirugía laparoscópica está establecida como de elección para el tratamento ablativo de la mayoría de las patologías del riñón y la glándula suprarrenal. Para las cirugías reconstrutivas, cuya curva de aprendizaje es mayor, esta técnica debe considerarse una opción viable pero en manos mas entrenadas $(10,11)$.

El acceso extraperitoneal es la vía habitual, familiar y clasicamente consagrada cuando se utiliza la cirugía abierta convencional en el tratamiento de las enfermedades urológicas. Sus ventajas incluyen la no manipulación de vísceras abdominales, evitando lesiones directas o por separación; la restricción de drenaje de eventuales secreciones (especialmente de orina) al espacio extraperitoneal, direccionando posíbles fístulas y reduciendo cuadros de irritación peritoneal; la aparición poco frecuente de íleo paralítico post-operatorio; la escasa o nula formación de adherencias intraabdominales tardías; y la incidencia significativamente menor de herniaciones por las incisiones quirúrgicas (12-14).

Algunos grupos en los Estados Unidos, Francia, Alemania, India y Japón vienen utilizando preferencialmente el acceso extraperitoneal en virtud de las razones expuestas anteriormente. Sin embargo, todavía es pequeña la proporción de cirugías realizadas por este acceso $(1-3,10,11,15)$.

La mayoría de los trabajos comparativos, entre los accesos transperitoneal y extraperitoneal han 
mostrado equivalencia terapéutica y de complicaciones, con algunos relatando el tiempo quirúrgico menor para la vía extraperitoneal $(2,3,16,17)$.

Existen algunos factores que pueden dificulta $r$ o imposibilitar el empleo del acceso extraperitoneal. Condiciones donde no hay capacidad para creación del espacio entre el riñón y la musculatura abdominal, como en los casos de cirugía previa, intensa inflamación renal y la presencia de riñones muy grandes son contraindicaciones relativas $(15,16)$.

Consideramos que la biopsia renal es el modelo ideal para la adaptación a la técnica y familiarización de la anatomía quirúrgica laparoscópica en las cirugías lumbares, permitiendo que se adquiera seguridad suficiente para iniciar procedimientos mas complejos (5).

En los 12 casos descritos de nefrectomía laparoscópica para enfermedades renales inflamatorias, 9 de ellos con tamaño renal de hasta $12 \mathrm{~cm}$ fueron realizados a través de acceso extraperitoneal exclusivo. El tiempo quirúrgico promedio fue superior a los casos operados sin inflamación local, sin embargo no hubo necesidad de conversión en esta serie. La nefrectomía laparoscópica para enfermedad renal inflamatoría es una cirugía de alto grado de complejidad, con índice de conversión más elevado, pero impone una recuperación mas rápida a los pacientes (18). Por lo tanto, debe ser considerada como una opción terapéutica y prácticada por laparoscopistas con experiencia.

Para la nefrectomía radical, el acceso retroperitoneoscópico evidenció una opción extremamente interesante. Una ventaja significativa, a pesar que su empleo es cuestionado, es el abordaje precoz del pedículo renal con inmediata ligadura de la arteria renal, maniobra que es habitualmente más difícil cuando se utiliza el acceso transperitoneal. Fueron operados tumores hasta de $10 \mathrm{~cm}$ en el mayor eje, no presentando dificultad técnica adicional en los tumores de mayores dimensiones. El tiempo quirúrgico y la tasa de complicaciones relatadas en este estudio estan de acuerdo con los mejores resultados de la literatura $(11,19)$.

Con relación a la cirugía de la adrenal, el acceso extraperitoneal fue factíble y de fácil aprendizaje. Los resultados nuestros de un trabajo comparativo entre los accesos trans y extraperitoneal mostraron que este permitió un tiempo quirúrgico menor, la realimentación más precoz y no hubo lesiones viscerales periféricas.

$\mathrm{N}$ inguna de las complicaciones obtenidas fueron directamente relacionadas con el acceso extraperitoneal. En todos los casos donde hubo drenaje de secreciones, no se observó irritación peritoneal, factor este traducido por el tiempo reducido de íleo paralítico y la rápida realimentación oral. En este particular, algunas series han demostrado ventajas para el acceso extraperitoneal $(3,17)$.

El índice de conversión fue menor de 1\%, ocurriendo apenas una al início de la experiencia motivado a la dificuldad en la identificación anatómica, certificando que el aprendizaje no es tan complejo por lo descrito anteriormente. La ocurrencia de lesión visceral por maniobras de separación o por corriente térmica fue nula. Las complicaciones que se presentan con el acceso transperitoneal, a pesar de raras, son frecuentemente mas graves, muchas veces necesitando de reintervención quirúrgica (17). N uestra impresión es que las complicaciones de la cirurgía laparoscópica extraperitoneal son de menor gravedad y generalmente pueden ser resueltas conservadoramente, sin la necesidad de abordaje quirúrgico adicional.

El gran desafío de este acceso son las cirugías reconstructivas (20). Además de depender de la habilidad individual y mucho entrenamiento, el planea miento correcto y la angulación adecuada de los trócares son fundamentales para operar con menor dificuldad en una cámara de trabajo que es más restringida. La manipulación de la aguja y de la sutura también causan limitaciones adicionales. Eventualmente, una posición no confortable de el cirujano para la ejecución de los puntos, puede agregar dificultades al procedimiento. Creemos que un contínuo entrenamiento tiende a suplantar estas limitaciones.

De igual manera para aquellos que prefiren la vía transperitoneal, el conocimiento del acceso extraperitoneal puede ser extremadamente útil cuando existe antecedentes de cirugía abdominal o peritonitis previa, situaciones en que las adherencias intraperitoneales pueden por si solas imposibilitar la cirugía.

Sabemos, de manera general, que la mayoría de los estudios todavía no arrojan ventajas significati- 
vas al abordaje laparoscópico extraperitoneal sobre la transperitoneal, la escogencia del acceso es basada esencialmente en la preferencia de el cirujano y las particularidades de cada caso.

\section{CONCLUSIONES}

El acceso extraperitoneal es la vía habitual para el abordaje de las enfermedades del tracto urinario, siendo ampliamente familiar al urológo.

Las principales ventajas atribuidas al acceso extraperitoneal laparoscópico son la no violación del peritoneo, manteniendo distante las vísceras intraperitoneales, con menor riesgo de formación de adherencias. La frecuencia de íleo post-operatorio es mínima. El acceso y control del hilio renal puede ser mas rápido y precoz. Una eventual fístula urinaria presenta evolución espontánea y benigna, sin riesgo de contacto con las estructuras intra-abdominales y sin íleo paralítico prolongado.

La cirugía laparoscópica extraperitoneal es una alternativa técnica útil y factible para el abordaje de varias patologías del riñón, ureter y adrenal. La adaptación espacial, inicialmente dificultada por la menor disponibilidad de parámetros ana tómicos y por la menor cámara de trabajo, puede ser rápidamente adquirida con entrenamiento. Los procedimientos reconstructivos son factíbles, pero técnicamente mas difíciles que los ablativos.

Dadas las ventajas y limitaciones discutidas previamente, nuestro servicio utiliza prioritariamente el abordaje extraperitoneal como acceso preferencial en la mayoría de los procedimientos laparoscópicos de los riñones y de las adrenales.

\section{BIBUOGRAFIA y LECTURAS RECOMENDADAS (*lectura de interés y **lectura fundamental)}

*1. GILL, I.S.; RASSWEILER, J.J.: "Retroperitoneoscopic renal surgery: our approach". Urology, 54: 734, 1999.

**2. GILL, I.S.; CLAYMAN, R.V.; ALBALA, D.M. y cols.: "Retroperitoneal and pelvic extraperitoneal laparoscopy: an international perspective". Urology, 52: 566, 1998.

**3. SUZUKI, K.; KAGEYAMA, S.; HIRANO, Y. y cols.: "Comparison of 3 surgical approaches to laparoscopic adrenalectomy: a nonrandomized, background matched analysis". J. Urol., 166: 437, 2001.

*4. TOBIAS-MACHADO, M.; JULIANO, R.V.; GASPAR, H.A. y cols.: "Videoendoscopic surgery by extraperitoneal access: technical aspects and indication". Int. Braz. J. Urol., 29: 441, 2003.

5. TOBIAS-MACHADO, M.; PINTO, M.A.; JULIANO, R.V. y cols.: "Retroperitoneoscopic renal biopsy". Braz. J. Urol., 28:192, 2002.

6. TOBIAS-MACHADO, M.; PINTO, M.A.; JULIANO, R.V. y cols.: "Preliminary experience with ureteral intussuseption in exclusive retroperitoneoscopic nephroureterectomy: a simple and safe option for the ressection of distal ureter and bladder cuff'. Arch. Esp. Urol., 55: 582, 2002.

7. TOBIAS-MACHADO, M.; PINTO, M.A.; JULIANO, R.V. y cols.: "Alternatives for distal ureter ressection in laparoscopic nephroureterectomy". Braz. J. Urol., 28: 109, 2002.

8. TOBIAS-MACHADO, M.; CARTUM, J.; SANTOSMACHADO, T.M. y cols.: "Retroperitoneoscopic adrenalectomy in an infant with adrenocortical virilizing tumor”. São Paulo Med. J., 120: 87, 2002.

*9. GAUR, D.D.: "Laparoscopic operative retroperitoneoscopy: use of a new device". J. Urol., 148: 1137, 1992.

**10. RASSWEILER, J.J.; SEEMANN, O.; FREDE, T. y cols.: "Retroperitoneoscopy: experience with 200 cases". J .Urol., 160: 1265, 1998.

11. ABBOU, C.C.; CICCO, A.; GASWAN, D.: "Retroperitoneal laparoscopic versus open radical nephrectomy". J. Urol., 161: 1776, 1999.

*12. KUMAR, M.; KUMAR, R.; HEMAL, A.K. y cols.: "Complications of retroperitoneoscopic surgery at one center". BJU Int., 87: 607, 2001.

13. SHANBERG, A.M.; SANDERSON, K.; RAJPOOT, D. y cols.: "Laparoscopic retroperitoneal renal and adrenal surgery in children". BJU Int., 87: 521, 2001.

*14. MERANEY, A.M.; SAMEE, A.A.; GILL, I.S.: "Vascular and bowel complications during retroperitoneal laparoscopic surgery". J. Urol., 168:1941, 2002.

**15. HEMAL, A.K.; ARON, M.; GUPTA, N.P. y cols.: "The role of retroperitoneoscopy in the management of renal and adrenal pathology". BJU Int., 83: 929, 1999.

16. HEMAL, A.K.; WADHWA, S.N.; KUMAR, M. y cols.: "Transperitoneal and retroperitoneal laparoscopic nephrectomy for giant hydronephrosis". J. Urol., 162: 35, 1999.

17. VAllancien, G.; CATHElineaU, X.; BAUMERT, H. y cols.: "Complications of transperitoneal laparoscopic surgery in urology: review of 1,311 procedures at a single center". J. Urol., 168: 23, 2002.

18. SIQUEIRA, T.M. Jr.; KUO, R.L.; GARDNER, T.A. y cols.: "Major complications in 213 laparoscopic nephrectomy cases: the Indianapolis experience". J. Urol., 168: 1361, 2002.

**19. GILL, I.S.; SCHWEIZER, D.; HOBART, M.G. y cols.: "Retroperitoneal laparoscopic radical nephrectomy: the Cleveland Clinic experience". J. Urol., 163: $1665,2000$.

*20. JANETSCHEK, G.; PESCHEL, R.; ALTARAC, S. y cols.: "Laparoscopic and retroperitoneoscopic repair of ureteropelvic junction obstruction". Urology, 47: 311, 1996. 